\title{
Les porphyries héréditaires: de la pathologie moléculaire à la thérapie génique
}

\begin{abstract}
Depuis la publication d'une synthèse dans médecine/sciences en 1987 sur les porphyries héréditaires [1], nos connaissances sur les gènes de la biosynthèse de l'hème et leur régulation ainsi que sur les mutations responsables des porphyries ont largement progressé. De plus, deux aspects nouveaux que sont l'étude des modèles animaux et les premiers essais de transfert de gènes en vue de thérapie génique ont commencé à se développer.
\end{abstract}

Progrès effectués dans la connaissance des gènes de la biosynthèse de l'hème

Sept des huit gènes codant pour les enzymes de la synthèse de l'hème ont été clonés, le plus récent étant celui de la coproporphyrinogène oxydase [2, 3] (Tableau I). Le dernier gène "récalcitrant" est celui de la protoporphyrinogène oxydase, qui a été caractérisé chez $B$. subtilis et $S$. cerevisiae mais ne l'est pas encore chez l'homme.

Ces gènes sont tous uniques, à l'exception de celui codant pour l'aminolévulinate (ALA) synthase pour laquelle deux gènes distincts ont été caractérisés. Ces différents gènes ne sont pas regroupés mais se trouvent au contraire entièrement dispersés sur tout le génome (Tableau I). Leur expression coordonnée au cours de la différenciation érythropoïétique doit donc être sous la dépendance de gènes de régulation codant pour des facteurs transcriptionnels qui se fixent sur les régions régulatrices.

L'organisation génétique de ces gènes est très variable. La première enzyme,
l'ALA synthase, est codée par deux gènes distincts, un gène d'expression ubiquitaire et un gène spécifiquement exprimé dans le système érythropoïétique. Les deux enzymes suivantes, l'ALA déshydratase et la porphobilinogène (PBG) désaminase sont chacune codées par un gène unique possédant deux promoteurs (l'un ubiquitaire, l'autre spécifique de la différenciation érythropoiétique). Les trois enzymes suivantes (l'uroporphyrinogène III synthase, l'uroporphyrinogène décarboxylase, la coproporphyrinogène oxydase), ainsi que la dernière (ferrochélatase) sont exprimées sous le contrôle d'un gène unique possédant un seul promoteur. A noter que cette dernière enzyme possède deux ARNm qui diffèrent dans leur partie 3' non codante par l'utilisation de deux signaux de polyadénylation différents. L'activité transcriptionnelle de ces gènes augmente de façon coordonnée au cours de la différenciation érythropoïétique [4].

Le promoteur du gène codant pour l'ALA synthase spécifique de la différenciation érythropoiétique (ALAS1), les promoteurs érythropoiétiques des gènes codant pour l'ALA déshydratase et la PBG désaminase, ainsi que les promoteurs des autres gènes connus, possèdent des éléments actifs en cis reconnus par des facteurs transcriptionnels spécifiques de l'érythropoïèse et ubiquitaires (association de motifs GATA/NFE2/CCACC/CCGCC) ([5] et $m / s n^{\circ} 4$, vol. $7, p$. 385; $n^{\circ} 11$, vol. 10, p. 1174).

L'ALA synthase joue un rôle central dans la régulation de la synthèse de l'hème tant au niveau du foie que du système érythropoïétique. Dans le foie, elle en est l'étape limitante à l'état basal et est fortement induite sous l'action de nombreuses substances liposolubles endogènes et exogènes. L'hème, produit final, exerce un rétrocontrôle négatif sur la synthèse et la maturation de l'enzyme (figure 1). Au niveau du système érythropoïétique, il existe une régulation coordonnée de la synthèse de l'hème et du métabolisme du fer par l'intermédiaire des éléments de réponse au fer (IRE, iron response element) $\left(\mathrm{m} / \mathrm{s} n^{\circ} 10\right.$, vol. 9, p. 1145), [6]. Dans les conditions où les apports en fer sont limités, la traduction des ARN messagers de l'ALA synthase érythropoïétique et de la ferritine est réprimée et les ARNm des récepteurs de la transferrine sont stabilisés, permettant d'éviter une accumulation de porphyrines lorsqu'il n'y a pas assez de fer pour former l'hème (figure 2). L'hème, en retour, contrôle la captation du fer, soit en inhibant l'endocytose des complexes fer-transferrine-récepteur, soit en inhibant la dissociation du fer de sa liaison à la transferrine après l'internalisation du complexe.

\section{Pathologie moléculaire de la biosyn- thèse de l'hème}

Durant ces dernières années, le clonage de différentes enzymes de la voie de biosynthèse de l'hème a permis la caractérisation des anomalies moléculaires responsables du déficit enzymatique chez de nombreux patients.

- Les mutations du gène codant pour l'ALA synthase érythropoïétique sont responsables de formes familiales d'anémie sidéroblastique de transmission récessive liée au chromosome X. Chez certains patients, l'anémie peut être corrigée par des doses 
Tableau I

LES ENZYMES ET GĖNES CORRESPONDANTS DE LA BIOSYNTHĖSE DE L'HĖME

\begin{tabular}{|c|c|c|c|c|}
\hline Enzyme & Symbole & $\begin{array}{c}\text { Taille } \\
\text { du gène }\end{array}$ & $\begin{array}{l}\text { Taille de l'ARNm } \\
\text { (cadre de } \\
\text { lecture ouverte) }\end{array}$ & $\begin{array}{l}\text { Localisation } \\
\text { chromosomique }\end{array}$ \\
\hline (1) $\delta$-aminolévulinate synthase & $\begin{array}{l}\text { ALASI } \\
\text { ALAS2 }\end{array}$ & $22 \mathrm{~kb}, 11$ exons & $\begin{array}{l}1920 \\
1746\end{array}$ & $\begin{array}{r}3 p 21 \\
\times p 11.21\end{array}$ \\
\hline $\begin{array}{l}\text { (2) } \delta \text {-aminolévulinate déshydratase } \\
\text { ou } \\
\text { porphobilinogène synthase }\end{array}$ & $\begin{array}{l}\text { PBGS } \\
\text { (ALAD) }\end{array}$ & & 990 & $9 q 34$ \\
\hline $\begin{array}{l}\text { (3) Porphobilinogène désaminase } \\
\text { ou } \\
\text { hydroxyméthylbilane synthase }\end{array}$ & $\begin{array}{l}\text { HMBS } \\
\text { (PBGD) }\end{array}$ & $10 \mathrm{~kb}, 15$ exons & $\begin{array}{l}1032 \\
1083\end{array}$ & $11 q 24$ \\
\hline (4) Uroporphyrinogène III synthase & UROS & $45 \mathrm{~kb}$ & 795 & $10 q 25-q 26$ \\
\hline (5) Uroporphyrinogène décarboxylase & UROD & $3 \mathrm{~kb}, 10$ exons & 1101 & $1 p 34$ \\
\hline (6) Coproporphyrinogène oxydase & COPROX & $14 \mathrm{~kb}$ & 1362 & $3 q 12$ \\
\hline (7) Protoporphyrinogène oxydase & PROTOX & NC & NC & 14 \\
\hline (8) Ferrochélatase & FC & $45 \mathrm{~kb}, 11$ exons & 1269 & $18 q 21,3$ \\
\hline
\end{tabular}

$N C$ : non connue.

Figure 1. Régulation classique de la biosynthèse de l'hème par le produit final. La régulation de la synthèse de I'hème a lieu essentiellement au niveau de I'ALA synthase, première enzyme de la chaîne de biosynthèse: le produit final exerce un rétrocontrôle négatif sur la synthèse et/ou la maturation de l'enzyme, aussi bien dans le foie que dans le système érythropoiétique. ALA: acide $\delta$-aminolévulinique. $P B G$ : porphobilinogène; HMB: hydroxyméthylbilane. Le nom des différentes enzymes (numérotées de 1 à 8) se trouve dans le Tableau I.

pharmacologiques de pyridoxine, le phosphate de pyridoxal étant le coenzyme de l'ALA synthase. L'identification récente de plusieurs mutations du gène de l'AL.A synthase indique que certaines de ces mutations entraînent une diminution d'affinité de l'enzyme pour le phosphate de pyridoxal chez des patients sensibles à la pyridoxine $[7,8]$.

pour les enzymes situées en aval de I'ALA synthase sont responsables des porphyries héréditaires. Selon les porphyries, les conséquences métaboliques du blocage enzymatique prédominent dans le foie ou dans les cellules érythropoïétiques. Quelle que soit la porphyrie considérée, les mutations responsables de la maladie sont extrêmement hétérogènes (une centaine connue pour la porphyrie la plus fréquente, la porphyrie aiguë intermittente [9], une douzaine connue pour la porphyrie la plus rare, la porphyrie érythropoiétique congénitale [10]). Certaines mutations sont néanmoins largement prédominantes, facilitant le diagnostic génotypique (cas de la porphyrie érythropoiétique congénitale ou de la porphyrie hépatoérythrocytaire où une mutation spécifique est retrou- 


\section{A $\cdot$ Faible concentration en Fer}
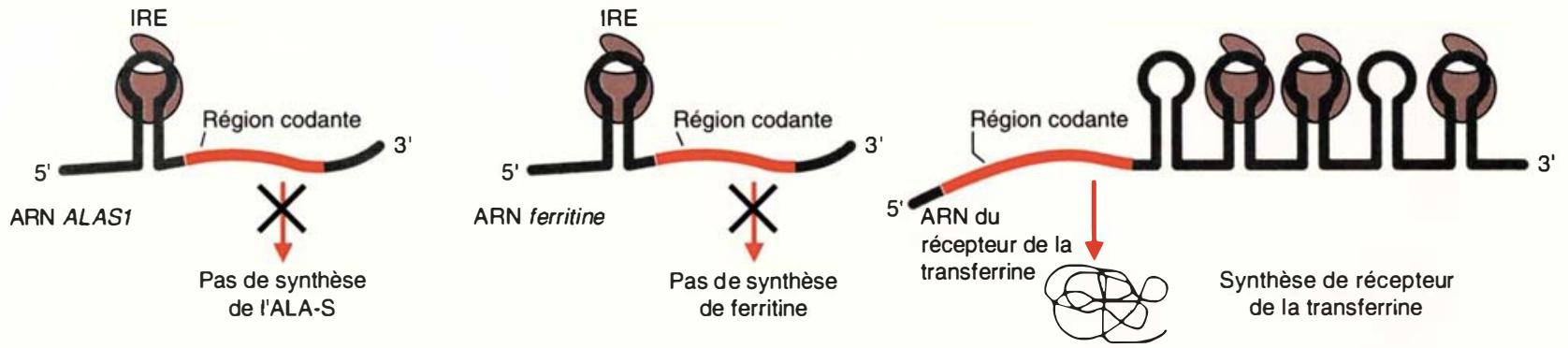

B • Forte concentration en Fer
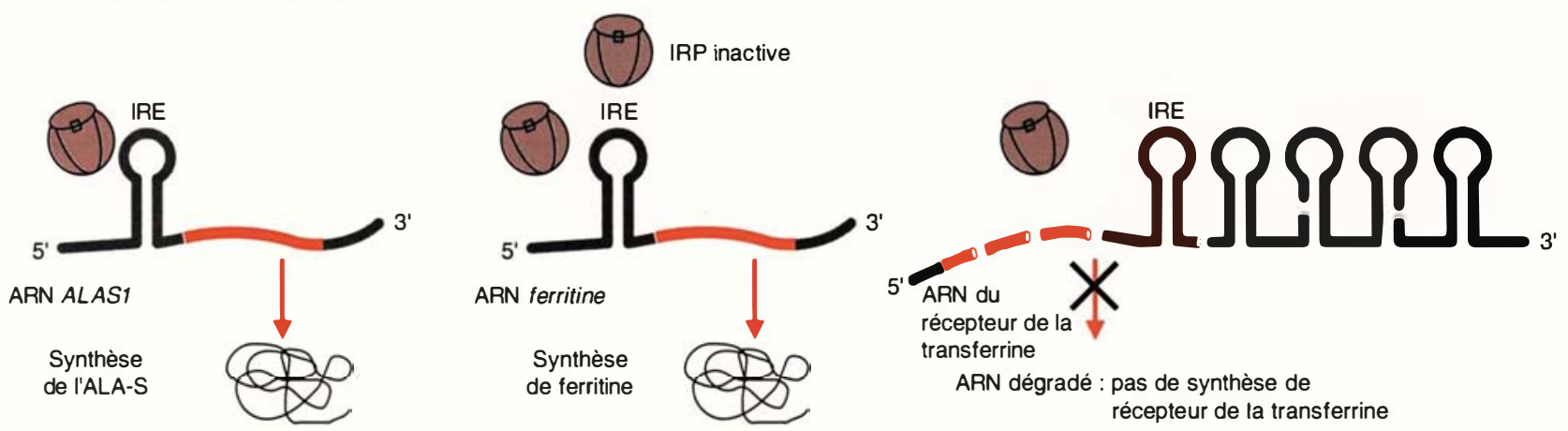

Figure 2. Régulation traductionnelle par le fer de l'ALA synthase dans le système érythropoiétique. L'ARNm du gène ALAS1 possède dans sa partie $5^{\prime}$ non codante un élément de réponse au fer (IRE) tout à fait comparable à celui de I'ARNm ferritine, mais qui ne se retrouve pas dans l'ARNm du gène d'expression ubiquitaire ALAS2. A. Lorsque le niveau intracellulaire du fer est faible, une protéine régulatrice dénommée IRP (iron responsive protein) se fixe à l'IRE et bloque la traduction des messagers ALAS 1 et ferritine. B. Quand la concentration en fer est élevée, la protéine IRP est inactive et les messagers ALAS1 et ferritine sont traduits. Dans le même temps, l'ARNm du récepteur de la transferrine est dégradé, limitant ainsi la capture du fer par la cellule. Il existe donc une régulation coordonnée de la capture, du stockage et de l'utilisation du fer dans les cellules érythropoiétiques.

vée sur environ $50 \%$ des allèles mutés). D’une manière générale, une liaison génotype/phénotype est retrouvée dans le cas des porphyries de transmission récessive, alors qu'une absence de liaison est la règle pour les porphyries de transmission dominante comme la porphyrie aiguë intermittente [9].

La possibilité de suivre les mutations par l'étude de l'ADN dans les familles est une aide précieuse pour le diagnostic des porteurs de la maladie. L'enquête familiale, indispensable en particulier pour la prévention des crises aiguës dans les porphyries hépatiques graves ou, dans le cadre d'un conseil génétique, pour les porphyries érythropoïétiques très sévères, est réalisée actuellement par les techniques de la biologie moléculaire qui complètent efficacement les études enzymatiques.

\section{Les modèles animaux de porphyries}

Deux porphyries existent à l'état naturel chez l'animal: la porphyrie érythropoiétique congénitale (PEC) et la protoporphyrie érythropoiétique (PPE). Ces animaux porphyriques ont été décrits respectivement chez le bœuf, le porc et l'écureuil pour la PEC et chez le bœuf pour la PPE (Tableau II). Il ne s'agit pas d'ani- maux couramment utilisés dans les laboratoires: aussi plusieurs modèles animaux murins viennent d'être créés ou sont en cours de réalisation. Une souris déficitaire en ferrochélatase a été obtenue en 1990 dans le laboratoire de J.-L. Guénet, à l'Institut Pasteur, après mutagenèse chimique par l'éthylnitrosourée [11]. Une mutation ponctuelle dans un exon du gène de la ferrochélatase est responsable d'un déficit enzymatique profond [12]. Le phénotype des souris $\mathrm{Fech}^{\text {mIlPas }} / \mathrm{Fech}^{m ! l \text { Pas }}$ reproduit assez fidèlement la forme grave de la maladie humaine: photosensibilité cutanée, hémolyse, cholestase due à l'accumulation de protoporphyrine 
Tableau II

CLASSIFICATION DES PORPHYRIES HUMAINES ET MODĖLES ANIMAUX CORRESPONDANTS

\begin{tabular}{|c|c|c|c|}
\hline Classification & Enzyme déficitaire & Transmission & Modèles animaux \\
\hline $\begin{array}{l}\text { Porphyries érythropoïétiques } \\
\text { - Porphyrie érythropoïétique congénitale } \\
\text { - Protoporphyrie érythropoïétique } \\
\text { - Porphyrie hépatoérythrocytaire } \\
\text { Porphyries hépatiques } \\
\text { - Formes aiguës } \\
\text { - Porphyrie aiguë intermittente } \\
\text { - Coprophorphyrie héréditaire } \\
\text { - Porphyrie variegata } \\
\text { - Porphyrie cutanée tardive (familiale) }\end{array}$ & $\begin{array}{l}\text { Uroporphyrinogène III synthase } \\
\text { Ferrochélatase } \\
\text { Uroporphyrinogène décarboxylase } \\
\text { Porphobilinogène désaminase } \\
\text { Coproporphyrinogène oxydase } \\
\text { Protoporphyrinogène oxydase } \\
\text { Uroporphyrinogène décarboxylase }\end{array}$ & $\begin{array}{l}\text { Récessive } \\
\text { Dominante } \\
\text { ou récessive } \\
\text { Récessive }\end{array}$ & $\begin{array}{l}\text { Bœuf, porc, } \\
\text { écureuil, souris }{ }^{c} \\
\text { Bœuf, souris }^{a} \\
\text { Souris }^{c}\end{array}$ \\
\hline
\end{tabular}

(a) Modèle animal obtenu par mutagenèse chimique. Modèle animal obtenu (b) ou en cours d'obtention (c) par recombinaison homologue dans les cellules ES.

dans le foie, ictère, fibrose hépatique (figure 3).

Plus récemment, un modèle animal de porphyrie aiguë a été créé par recombinaison homologue dans les cellules ES puis injection de ces cellules dans un blastocyste de souris [13]. Les auteurs ont délété partiellement le gène porphobilinogène désaminase dans ces cellules par insertion du gène $n e o^{R}$. Les animaux hétérozygotes ont les caractéristiques biochimiques de la maladie, à savoir augmentation d'excrétion d'acide $\delta$ aminolévulinique et porphobilinogène. Ce modèle pourra être intéressant pour la compréhension des anomalies neurologiques observées au cours des crises aiguës de porphyrie. Enfin, deux autres modèles murins de porphyries sont en cours d'élaboration: un modèle de porphyrie hépatoérythrocytaire (laboratoire d'hématologie, J. Kushner, Salt Lake City, USA) et un modèle de porphyrie érythropoïétique congénitale (laboratoire de biochimie et biologie moléculaire, H. de Verneuil, université de Bordeaux-II).

\section{Le besoin d'une thérapie génique} somatique dans les porphyries

Le traitement des porphyries est dif876 porphyries hépatiques aiguës (Tableau II) bénéficient d'un traitement symptomatique ainsi que d'un traitement spécifique, à savoir l'hème-arginate (Normosang ${ }^{\circledR}$ ) qui joue un rôle répresseur sur l'ALAsynthase [1]. Insistons aussi sur l'importance de la prévention des crises aiguës en évitant la prise ou l'administration de médicaments "porphyrinogènes " grâce à la détection des porteurs asymptomatiques (études enzymatiques et/ou analyse des mutations au niveau de l'ADN). La porphyrie cutanée tardive est une maladie sans gravité qui peut être stabilisée facilement par un traitement symptomatique (essentiellement les séries de saignées ou la prise de chloroquine à faibles doses). En revanche, les formes sévères des porphyries érythropoḯtiques (porphyrie érythropoïétique congénitale ou PEC ; porphyrie hépatoérythrocytaire ou PHE, formes graves de protoporphyrie érythropoiétique avec complications hépatiques) ne bénéficient d'aucune thérapeutique efficace et, en tant que maladies génétiques, sont naturellement candidates à une thérapie génique. Les différentes questions à résoudre pour cette approche thérapeutique sont: (1) Quel(s) est (sont) le(s) tissu(s) cible(s) à atteindre? A-t-on besoin d'une expression spécifique de tissu et d'une régulation fine de cette expression? (2) Quel système de transfert (viral ou non viral) et quel mode d'administration (in vivo ou ex vivo) peuvent être envisagés? (3) Quelle est l'efficacité minimale requise (pourcentage de cellules transduites) et quel niveau d'expression doit-on atteindre pour avoir une correction du phénotype "porphyrique"? Nous essaicrons de répondre à ces questions en prenant comme modèle la porphyrie érythropoiétique congénitale et en commentant les premiers résultats obtenus in vitro dans cette maladie.

La porphyrie érythropoïétique congénitale, maladie hématologique candidate à une thérapie génique

L'expression du déficit enzymatique dans cette maladie se situe essentiellement au niveau du système érythropoiétique, justifiant les tentatives de transplantation médullaire dans l'enfance à partir de donneurs identiques dans le système HLA. Un seul cas de patient traité a été rapporté montrant une amélioration très nette de la maladie, mais celui-ci est décédé onze mois plus tard d'une infection à cytomégalovirus [14]. Cette thérapie cellulaire ne pouvant être 

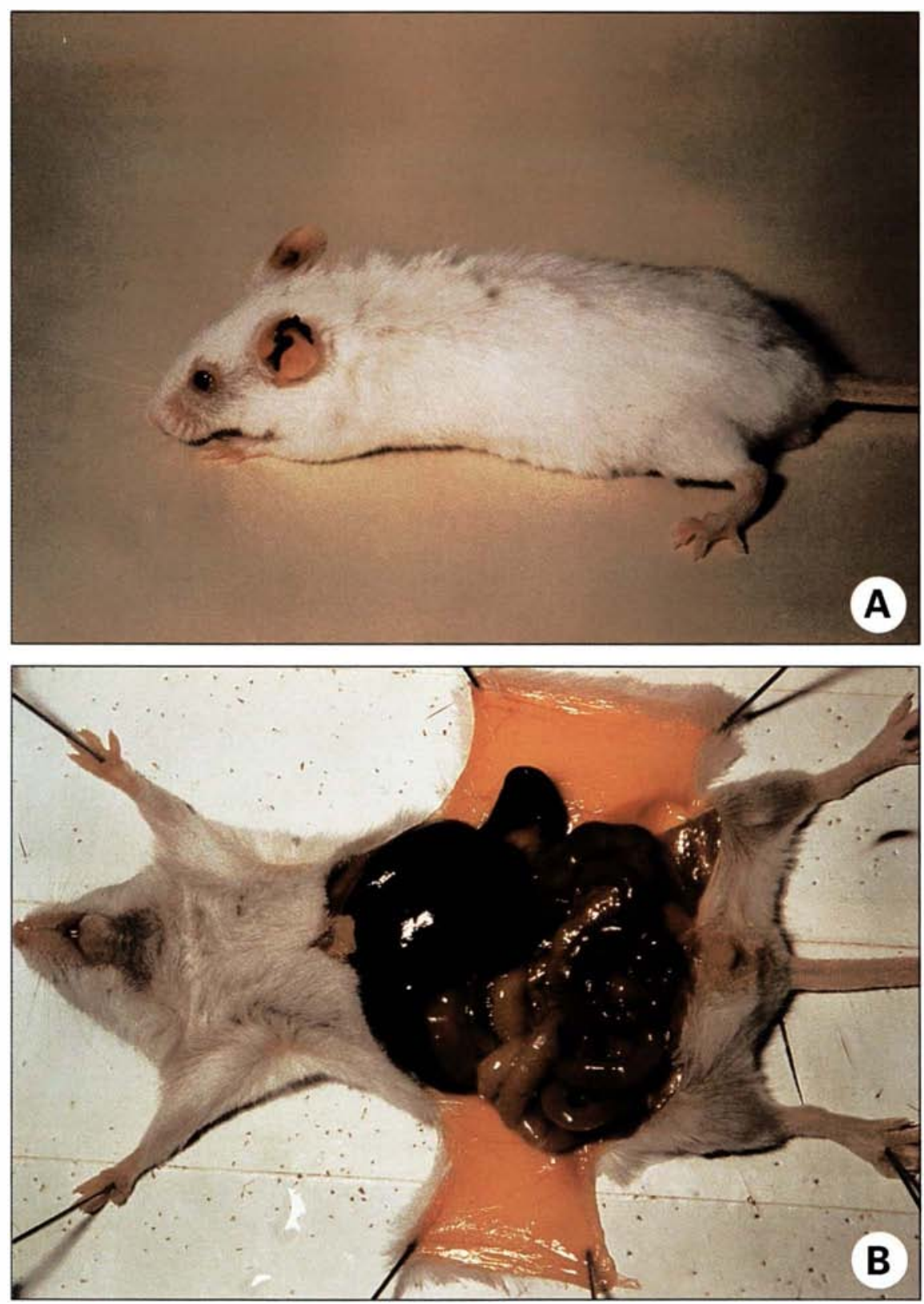

Figure 3. La souris protoporphyrique Fechm1Pas/Fechm1Pas A. La photosensibilité est manifeste après exposition au rayonnement UV: des lésions inflammatoires devenant éventuellement ulcéreuses apparaissent au niveau des oreilles, du museau et du dos de l'animal. B. Le foie et la rate sont très augmentés de volume et apparaissent de couleur foncée, en relation avec l'accumulation très importante de protoporphyrine dans ces organes. L'ictère est manifeste au niveau du tissu sous-cutané et du péritoine. (Clichés aimablement fournis par X. Montagutelli et J.L. Guénet, Institut Pasteur, Paris).

réaliséc que dans $20 \%$ à $30 \%$ des cas, la thérapie génique des cellules hématopoiétiques est une alternative thérapeutique intéressante. Les systèmes viraux (rétrovirus et virus associé à l'adénovirus ou AAV) sont $m / s n^{\circ} 6, v o l .11$, juin 95

dans des fibroblastes humains déficients et avons obtenu une correction totale du déficit enzymatique dans ces cellules [16]. L'essai a été réalisé ensuite sur des lymphoblastes immortalisés (étant donné la difficulté d'obtention des cellules hématopoïétiques à partir de la moelle osseuse ou des cellules sanguines de patients): nous avons pu démontrer une restauration de l'activité enzymatique et une correction métabolique de la maladie: l'accumulation très importante des porphyrines dans les cellules déficientes en présence d'acide $\delta$-aminolévulinique retombe à un niveau normal dans les cellules transduites. En revanche, les expériences de coculture entre cellules déficientes et cellules normales ou cellules déficientes et cellules corrigées ont montré l'absence de correction métabolique croisée [17]. Ces résultats indiquent que le transfert de gènes doit être efficace plutôt quant au nombre de cellules transduites qu'au niveau d'expression atteint, de manière à réduire très fortement l'accumulation des porphyrines responsables des signes pathologiques. Ce problème pourrait être en partie résolu par un avantage sélectif des cellules corrigées par rapport aux cellules normales, mais ce point n'est pas démontré actuellement. Concernant la régulation de l'expression du gène greffé, contrairement à d'autres maladies génétiques hématologiques comme les thalassémies, cette maladie ne requiert pas de régulation fine : en effet, l'UROIIIS n'est pas une étape limitante de la biosynthèse de l'hème et sa surexpression ne peut avoir de conséquences pathologiques pour les cellules déficientes. Le promoteur utilisé actuellement est de type fort et ubiquitaire (LTR des virus MoMLV/MoMSV ou gène cellulaire de la phosphoglycérate kinase PGK). L'extinction à long terme in vivo de ce type de promoteur (qui n'est pas démontrée) pourrait conduire à l'utilisation d'un promoteur spécifique de la différenciation érythropoïétique du type LCR (locus control region) [18].

Le dernier point à considérer est la cellule cible idéale pour une thérapie génique à long terme: il s'agit, bien sûr, de la cellule souche pluripotente 
capable d'autorenouvellement et de différenciation [19]. Nous ne savons pas à l'heure actuelle isoler cette cellule souche pluripotente et a fortiori y transférer le gène d'intérêt pour une persistance définitive de l'expression du transgène. Nous retombons ici sur le même problème que celui des autres maladies génétiques hématologiques candidates à une thérapie génique: l'exemple le plus caractéris tique est celui du déficit immunitaire combiné sévère dû au déficit en adénosine désaminase [20,21]. Le succès de la thérapie génique à long terme dépend donc essentiellement des progrès à venir concernant la biologie des cellules hématopoïétiques, qu'elles soient d'origine médullaire ou d'origine périphérique [22]. La disponibilité future d'un modèle animal murin doit nous permettre de réaliser des expériences de greffe de moelle et de transfert de gène, réalisant ainsi une thérapie cellulaire ou génique chez l'animal. Ce modèle doit nous permettre de démontrer que la correction du déficit enzymatique au niveau de la moelle osseuse est suffisante pour guérir la maladie

\section{RÉFÉRENCES}

1. De Verneuil H, Grandchamp B, Deybach J, Beaumont C, Nordmann Y. Les porphyries héréditaires: de la biochimie clinique à la pathologie moléculaire. médecine/sciences 1987; 3: 157-63.

2. Martasek P, Camadro JM, Delfau-Larue MH, Dumas JB, Montagné II, de Verneuil H, Labbe P, Grandchamp B. Molecular cloning, sequencing, and functional expression of a cDNA encoding human coproporphyrinogen oxidase. Proc Natl Acad Sci USA 1994; 91 : 3024-8.

3. Delfau-Larue MH, Martasek P, Grandchamp B. Coproporphyrinogene oxidase: gene organization and description of a mutation leading to exon 6 skipping. Hum Mol Genet 1994; 3 : 1325-30.

4. Fujita $H$, Yamamoto $M$, Yamagami $T$, Hayashi N, Bishop TR, de Verneuil $\mathrm{H}$ Yoshinaga T, Shibahara S, Morimoto R, Sassa $S$. Sequential activation of genes for heme pathway enzymes during erythroid differentiation of mouse friend virus-transformed erythroleukemia cells. Biochim Biophys Acta 1991 ; 1090 : 311-6.

5. Mignotte V, Lemarchandel V, Roméo PH. GATA et Ets: deux familles de déterminants majeurs de la différenciation hémato-
6. Theil EC. Iron regulatory elements (IREs) : a family of mRNA non-coding sequences. Biochem J 1994; 304: 1-11.

7. Cox TC, Bottomley SS, Wiley JS, Bawden MI, Matthews CS, May BK. X-linked pyridoxine-responsive sideroblastic anemia due to a Thr $r^{388}$-to-Ser substitution in erythroid 5aminolevulinate synthase. $N$ Engl J Med $1994 ; 330: 675-9$.

8. Cotter PD, Rucknagel DL, Bishop DF. Xlinked sideroblastic anemia: identification of the mutation in the erythroid-specific $\delta$ aminolevulinate synthase gene (ALAS2) in the original family described by Cooley. Blood 1994; 84: 3915-24.

9. Deybach JC, Puy H. Porphobilinogen deaminase: gene structure and molecular defects. J Bioenerg Biomembr 1995 (sous presse)

10. Bensidhoum M, Ged C, Hombrados I, Moreau-Gaudry F, Hift RS, Meissner P, Sturrock $\mathrm{ED}$, de Verneuil $\mathrm{H}$. Identification of two new mutations in congenital erythropoietic porphyria. Eur J Hum Genet 1995 (sous presse).

11. Tutois S, Montagutelli X, Da Silva V, Jouault H, Rouyer-Fessard P, Leroy-Viard K, Guénet JL, Nordmann Y, Beuzard Y, Deybach JC. Erythropoietic protoporphyria in the house mouse. A recessive inherited ferrochelatase deficiency with anemia, photosensitivity and liver disease. J Clin Invest 1991 88: 1730-6.

12. Boulechfar S, Lamoril J, Montagutelli X, Guénet JL, Deybach JC, Nordmann Y, Dailey H, Grandchamp B, de Verneuil H. Ferrochelatase structural mutant $\left(\mathrm{Fech}^{m / P P_{1}}\right)$ in the house mouse. Genomics 1993; 16:645-8.

13. Lindberg RLP, Grandchamp B, Porcher C, Ledermann B, Bürki B, Meyer UA PBGD-knockout mouse as an animal model for human porphyria. European Sociely of Human Genetic, 26th annual meeting, 1994 $n^{\circ} 334$ (abstr).

14. Kauffman L, Evans DIK, Stevens RF, Weinkove C. Bone-marrow transplantation for congenital erythropoietic porphyria. Lancet 1991 ; 337 : 1510-1.

15. Nienhuis AW. Gene transfer into hematopoietic stem cells. Blood Cells 1994; 20: 141-9.

16. Moreau-Gaudry F, Ged C, Barbot C, Mazurier F, Boiron JM, Bensidhoum M, Reiffers J, de Verneuil H. Correction of the enzyme defect in cultured congenital erythropoietic porphyria disease cells by retrovirus-mediated gene transfer. Hum Gene Ther 1995; 6: 13-20.

17. Moreau-Gaudry F, Mazurier F, Bensidhoum M, Ged C, de Verneuil H. Metabolic correction of congenital erythropoietic porphyria by retrovirus-mediated gene transfer into Epstein-Barr virus-transformed B-cell lines. Blood 1995; 85 : 1449-53.

18. Labie D, Krishnamoorthy R, Du nouveau dans les séquences activatrices des gènes de globine (LCR). médecine/sciences $1992 ; 8: 255-8$.
19. Coulombel L, Vainchenker W. Les cellules souches hématopoiétiques. médecine/sciences $1995 ; 1: 13-6$.

20. Bordignon C, Servida P, Notarangelo LD, Nobili N, Ferrari G, Rossini S, Mavilio $F$, Ugazio AG. Long term immune reconstitution following human somatic cell gene therapy in two patients affected by $\mathrm{ADA}$ deficient SCID. Blood 1994; 84 (suppl 1) : 402a (abstr).

21. Hoogerbrugge PM, Beusechem VV, Valerio D, Moseley A, Harvey M, Fischer A, Debree M, Gaspar B, Morgan G, Levinsky R. Gene therapy in 3 children with adenosine deaminase deficiency. Blood 1994;84 (suppl 1) : 402a (abstr).

22. Chabannon C, Mannoni, P. Les cellules souches hématopoiétiques du sang périphérique chez l'homme. médecine/sciences 1995; 1:17-27.

\section{Hubert de Verneuil}

\section{Cécile Ged}

\section{François Moreau-Gaudry}

Laboratoire de biochimie médicale et biologie moléculaire, université de Bordeaux-II, 146, rue Léo-Saignat, 33076 Bordeaux Cedex, France.

\section{Bernard Grandchamp}

Laboratoire de génétique moléculaire, Inserm U. 409, faculté de médecine Xavier Bichat, 16, rue Henri-Huchard, 75018 Paris, France.

\section{Jean-Charles Deybach \\ Yves Nordmann}

Service de biochimie, hôpital Louis-Mourier, 178, rue des Renouillers, 92701 Colombes Cedex, France.

Dernière minute : l'ADN complémentaire de la protoporphyrinogène oxydase vient d'être cloné chez l'homme, achevant la connaissance au niveau moléculaire de l'ensemble des gènes impliqués dans la biosynthèse de l'hème. (Nishimura $K$, Taketani $S$, Inoshuki $\mathrm{H}$. Cloning of human cDNA for protoporphyrinogen oxidase by complementation in vivo of hemG mutant of Escherichia coli. J Biol Chem 1995 ; 270 : 8076-80).

\section{TIRÉS À PART}

H. de Verneuil. 